\title{
Clinical utility of CT in children with persistent focal chest abnormality
}

\section{To the Editors:}

We read with interest the article by LI et al. [1] on the contribution of computed tomography (CT) in determining aetiological diagnosis of noncystic fibrosis (CF) bronchiectasis, and in leading to a change in management. We carried out a prospective study in 50 children (26 males; median age: $7.45 \mathrm{yrs}$, range: 12 months to $17 \mathrm{yrs}$ ) with chronic cough for $>3$ months and persistent focal chest abnormality on at least two conventional chest radiographs (CCR) made at intervals of $\geqslant 3$ months. We aimed to assess what additional information CT (alone or combined with clinical findings and further diagnostic tests) provides, and to determine whether gaining such new information influences the clinical management.

Sweat test, leukocytes blood count and purified protein derivative test for tuberculosis, electrocardiogram and Doppler echocardiography were normal or negative in all cases. Seven patients were affected by primary ciliary dyskinesia (PCD), five had previously undergone oesophageal atresia repair, three had common variable immunodeficiency and two were affected by Down's syndrome. Ongoing treatment included regular daily chest physiotherapy in patients with $\mathrm{PCD}$, and periodic intravenous immunoglobulins (Igs) in children with common variable immunodeficiency.

CT scans were performed within 1 month from the last CCR. All patients were re-examined every 4 months by the paediatric pulmonologist who had requested the CT scan, with any further investigation planned depending on the result of the CT scan. Further diagnostic tests included: nasal brushing for ciliary structure; sweat test and CF gene mutation analysis; 24-h oesophageal pH-metry; serum Ig plus IgG subclass determination; and bronchoscopy. The definitive diagnosis was made after 12 months on the basis of the clinical course and the results of any additional diagnostic tests performed. At the end of the follow-up period, we compared both CCR and CT with the definitive diagnosis and with the clinical outcome, and evaluated whether or not the clinical management had been modified.

Ultimate diagnoses were: consolidation (31 patients; 60\%); bronchiectasis (12 patients; $24 \%$ ); congenital malformations of the lower respiratory tract (six patients; $12 \%$ ); and foreign body (FB) inhalation (one patient; 2\%). Of the 12 patients with bronchiectasis, one subsequently had evidence of common variable immunodeficiency, two of PCD and two of CF mutations $(\Delta \mathrm{F} 508 / 3849+10 \mathrm{~Kb} \quad \mathrm{C} \rightarrow \mathrm{T}$ in one case, and $\mathrm{N} 1303 \mathrm{~K} / \mathrm{D} 1152 \mathrm{H}$ in the other).

Following lung $\mathrm{CT}$, alone or combined with clinical findings and additional tests, the management was changed in 32 children (64\%), but remained unmodified in 18 cases (36\%;
Chi-squared $=6.76 ; \mathrm{p}=0.009 ;$ table 1$)$. Changes consisted of either medical therapies (preventive use of antibiotics in 12 cases with bronchiectasis and more intensive chest physiotherapy, i.e. three times $\cdot$ day $^{-1}$ in 10 patients with consolidation) or nonmedical treatment. These included the endoscopic removal of a small fragment of a plastic toy in the case with FB inhalation, and chest surgery in nine subjects (three with PCD and persistent consolidation who underwent lobectomy and six suspected of having lung malformation). The surgical findings of bronchogenic cyst (three patients), congenital cystic adenomatoid malformation (two patients) and extra-lobar sequestration (one patient) confirmed the results of the CT scan in cases suspected of intrathoracic malformations. In 18 out of 32 patients undergoing management changes (56\%), CT alone provided sufficient diagnostic information, whereas, in the remaining $14(44 \%)$, decisions about clinical management were reached, in addition to radiological data, on the basis of tests other than imaging, as well as of information obtained during the follow-up.

Lung CT scans provide superior anatomic definition of airways abnormalities [2, 3]. As in non-CF bronchiectasis [1], in children with persistent focal chest abnormality a fundamental question that should be addressed is whether or not chest CT significantly alters patient's care. Cases that benefit from chest CT alone include those in whom CCR fails to adequately explain the clinical course (e.g. children with bronchiectasis or congenital lung malformations) [4]. However, CT may also be beneficial when the diagnostic probability that guides decision derives, in addition to lung imaging, from the combined use of ancillary laboratory findings and the progress of clinical symptoms and signs over an established period of time.

\begin{tabular}{|c|c|c|c|}
\hline TABLE 1 & \multicolumn{3}{|c|}{$\begin{array}{l}\text { Definitive diagnosis and change of management } \\
\text { following computed tomography, alone or } \\
\text { combined with additional tests, in } 50 \text { children } \\
\text { with chronic cough and persistent focal chest } \\
\text { abnormality }\end{array}$} \\
\hline \multirow{2}{*}{\multicolumn{2}{|c|}{ Definitive diagnosis }} & \multicolumn{2}{|c|}{ Clinical management } \\
\hline & & Changed & Unchanged \\
\hline \multicolumn{2}{|c|}{ Bronchiectasis } & 12 & 0 \\
\hline \multicolumn{2}{|c|}{ Lung malformation } & 6 & 0 \\
\hline \multicolumn{2}{|c|}{ Foreign body inhalation } & 1 & 0 \\
\hline \multicolumn{2}{|c|}{ Consolidation } & 13 & 18 \\
\hline \multicolumn{2}{|c|}{ Total } & $32(64)$ & $18(36)$ \\
\hline
\end{tabular}


Evaluating the clinical utility of diagnostic tests in paediatric pulmonary medicine is not an easy task because only a few standard reference tests exist against which to compare testing strategies in selected cases (e.g. lung biopsy or bronchoalveolar lavage findings in interstitial lung disease or surgical confirmation in lung malformations) [5]. In patients with chronic cough due to persistent chest disease, a "yes" or "not" response is often insufficient to decide whether the test is worthwhile, since correct diagnosis often relies on a combination of clinical information plus laboratory results. In these cases, the improvement of the final outcome following computed tomography scan, whether alone or combined, can provide the best demonstration of the clinical utility of computed tomography.

\section{S. Montella*, M.V. Andreucci*, L. Greco*, \\ F. Barbarano*, S. De Stefano*, L. Brunese ${ }^{\#}$ and \\ F. Santamaria*}

Depts of *Paediatrics, and "Radiology, Federico II University, Naples, Italy.

\section{REFERENCES}

1 Li AM, Sonnappa S, Lex C, et al. Non-CF bronchiectasis: does knowing the aetiology lead to changes in management? Eur Respir J 2005; 26: 8-14.

2 Webb WR. High-resolution CT of the lung parenchyma. Radiol Clin North Am 1989; 27: 1085-1097.

3 Kuhn JP, Brody AS. High-resolution CT of pediatric lung disease. Radiol Clin North Am 2002; 40: 89-110.

4 de Jong PA, Muller NL, Parè PD, Coxson HO. Computed tomographic imaging of the airways: relationship to structure and function. Eur Respir $J$ 2005; 26: 140-152.

5 Borrill Z, Houghton C, Sullivan PJ, Sestini P. Retrospective analysis of evidence base for tests used in diagnosis and monitoring of disease in respiratory medicine. BMJ 2003; 327: $1136-1138$ 